\title{
Model of Paradoxical Signaling Regulated T-Cell Population Control for Design of Synthetic Circuits*
}

\author{
Michaëlle N. Mayalu ${ }^{1}$, Harman Mehta $^{2}$ and Richard M. Murray ${ }^{3}$
}

\begin{abstract}
Paradoxical signaling occurs when the same signaling molecule can trigger antagonistic cell functions. For example, T-Cells secret cytokine IL-2 which promotes T-Cell proliferation and also affects cell death. It has been shown that cells with this signaling capability have bi-stable population dynamics and can achieve identical levels of population homeostasis independent of initial cell concentrations. These capabilities are desirable in the context of synthetic population control circuits designed for application in therapeutic treatment of various diseases. It thus becomes important to understand the dependence of the cell system on the intracellular paradoxical components and to develop accurate models to provide insight into optimal design characteristics. Here, we create a model that integrates three IL-2 driven intracellular mechanisms that trigger 1) T-cell proliferation 2) T-cell apoptosis and 3) IL-2 production. Using this model, we are able to explore the internal mechanisms necessary for paradoxical signaling in T-Cells. It was shown that the intracellular mechanisms considered were sufficient to produce population dynamic characteristics of paradoxical signaling consistent with published systems level models and data. Furthermore, analysis of parameters revealed dependency of population homeostatic stability on the production and activation of the specific intracellular proteins considered.
\end{abstract}

\section{INTRODUCTION}

An important topic in synthetic biology is development of cell-cell communication modules that can regulate population size of cell communities. Current research with bacterial cells create homeostatic cell population densities by synthetically interfacing quorum-sensing behavior with programmed cell death [1]. This enhanced cell capability has implications in gastrointestinal diseases caused by loss or disruption of healthy bacteria in the gut [2]. In order to alleviate this issue, one could introduce an engineered version of the disrupted bacteria that could maintain a constant cell population in the midst of environmental perturbations. In mammalian cells, there is also an increased need for a synthetic population control circuit. Such research has implications in immunotherapies that modify and enhance

*This research is partially supported by the Defense Advanced Research Projects Agency (Agreement HR0011-17-2-0008). The content of the information does not necessarily reflect the position or the policy of the Government, and no official endorsement should be inferred.

${ }^{1}$ Michaëlle N. Mayalu is with the Division of Engineering and Applied Science, California Institute of Technology, 1200 E California Blvd, Pasadena, CA 91125, USA mmayalud caltech.edu

${ }^{2}$ Harman Mehta is with the Department of Biochemical Engineering and Biotechnology, Indian Institute of Technology, Delhi, India harmanmehta98@gmail.com

${ }^{3}$ Richard M. Murray is with the Division of Engineering and Applied Science and the Division of Biology and Biological Engineering, California Institute of Technology, 1200 E California Blvd, Pasadena, CA 91125, USA murray@cds.caltech.edu the normal capacity of the patient's immune cells[3] and in synthetic wound healing where engineered epithelial, endothelial and immune cells(among others) require robust population control modules.

Research surrounding synthetic population control circuits could lead to treatment of disease by moving beyond traditional approaches and allowing the development smart therapies, where the therapeutic agent can make decisions based on intercommunication between adjacent cells and the environment. One key issue in the development of the previously described synthetic population circuits is their sensitivity to sensing mutations. These mutations can cause overgrowth of the cell population [4]. Furthermore in the aforementioned applications, it could be necessary to have two homeostatic states such that the cell population could expand or collapse to a specified size when needed.

These concerns can be addressed using a paradoxical signaling-based population control mechanism. Paradoxical signaling occurs when the same quorum sensing molecule can affect antagonistic cell behaviors. As a result, the population dynamics have bistable characteristics. At quorum sensing molecule levels below a certain threshold, the population can be maintained at a stable low or off population state. Above a certain threshold the cell population expands to a stable high or on population state. Within the paradoxical circuit architecture, mutated cells that have lost quorum sensing capabilities will be stuck in the off state. Cells with the same mutation would have taken over in the nonparadoxical population circuit [5].

T-Cells exhibit paradoxical signaling naturally by secreting a cytokine IL-2 which promotes T-Cell proliferation [6] and also affects cell death [7]. In order to build a synthetic paradoxical signaling regulated population control circuit with optimal characteristics, it is beneficial to understand the natural T-cell system by modeling intracellular paradoxical components. This can help identify the reaction modules that can be formulated synthetically. Previous models have described the dynamic interaction of IL-2 and cell population without elucidation of intracellular biochemical reactions regulated by IL-2 [4], [8], [5]. These models provide a systems level understanding of the paradoxical signaling population control system. Furthermore, they reveal bistable population dynamics as a consequence of IL-2 having a cooperative affect on proliferation rate and linear effect on death rate. This causes the net growth (proliferation minus death rate) as a function of cytokine IL-2 to be at equilibrium for three points. When the lowest and highest equilibrium points are stable, and the middle point unstable, paridoxical 
TABLE I

SUMMARY OF SPECIES INVOLVED IN INTRACELLULAR MECHANISTIC REACTION

\begin{tabular}{lc}
\hline Description & Species \\
\hline IL-2 cytokine & $\mathrm{I}$ \\
IL-2 production gene & $\mathrm{G}_{\mathrm{I}}$ \\
IL-2 receptor & $\mathrm{R}$ \\
IL-2 receptor production gene & $\mathrm{G}_{\mathrm{R}}$ \\
IL-2/receptor production gene complex & $\mathrm{IG}_{\mathrm{R}}$ \\
IL-2/receptor complex & $\mathrm{IR}$ \\
STAT5 Transcription factor & $\mathrm{S}$ \\
Activated STAT5 & $\mathrm{S}^{*}$ \\
Activated STAT5-gene complex & $\mathrm{G}_{\mathrm{I}} \mathrm{S}^{*}$ \\
FasL & $\mathrm{F}$ \\
FasL production gene & $\mathrm{G}_{\mathrm{F}}$ \\
IL2/FasL production gene complex & $\mathrm{IG}_{\mathrm{F}}$ \\
Caspase8 & $\mathrm{C}$ \\
Activated Caspase8 & $\mathrm{C}^{*}$ \\
\hline
\end{tabular}

signaling can be acheived. The pertinent question for synthetic biological design is: What are the possible intracellular mechanisms that lead to these affects? Our analysis will complement the aforementioned works and attempt to address this question by providing a mechanistic understanding of how the IL-2 regulated intracellular pathways can lead to the observed system behaviors in T-Cells. With their identification, synthetic biologists will be able to fabricate equivalent functional mechanisms and integrate them into various cells into order to enhance or modify their behavior.

Specifically in this paper, we perform analysis of intracellular mechanisms that directly affect population homeostatic stability. Furthermore we are able to show that the concentration of key intracellular proteins are directly related to cell proliferation and death rates and are sufficient to explain the characteristic relationship between IL-2 and net growth rate. This is distinct from previous works because we draw from intracellular mechanisms that have been previously characterized individually, to explain the overall system behavior of paradoxical signaling in T-cells. We show that these mechanisms are sufficient to gain further insight into the circuit functionality and stability properties.

This paper is organized as follows: In Section II, we give an overview of the known intracellular mechanisms involved in IL-2 induced T-cell proliferation, death and IL2 production. In Section III we propose a set of coupled intracellular-population dynamic equations based on mathematical derivations of reactions from Section II. In Section IV we simplify the full set of dynamic equations based on the assumption of rapid intracellular reactions compared to cell division. In Section V, we analyze the resulting simplified model using computational simulation for varied parameters. We summarize the main findings of the paper discuss future work in Section VI.

\section{IdENTIFICATION OF IL-2 Regulated Mechanisms of Proliferation, DeATh AND IL-2 Production}

Table I summarizes the species involved in the mechanisms described in the subsequent sections. Table II summarizes the complete reactions for IL-2 induced cell proliferation, cell death and IL-2 production that were considered.
TABLE II

SumMary INTRACELlular MEChanistic REACtion

\begin{tabular}{|c|c|c|}
\hline Description & \multicolumn{2}{|c|}{ Reactions } \\
\hline IL-2 environmental source & $\emptyset$ & $\stackrel{\mathrm{I}_{0}}{\longrightarrow} \mathrm{I}$ \\
\hline constitutive production of IL-2 & $\mathrm{G}_{\mathrm{I}}$ & $\stackrel{N \cdot \lambda}{\longrightarrow} G_{I}+I$ \\
\hline IL-2/receptor binding & $\mathrm{I}+\mathrm{R}$ & $\underset{\mathrm{k}_{2}}{\stackrel{\mathrm{k}_{1}}{\rightleftharpoons}} \mathrm{IR}$ \\
\hline IL-2/receptor gene binding & $\mathrm{I}+\mathrm{G}_{\mathrm{R}}$ & $\underset{\mathrm{k}_{4}}{\stackrel{\mathrm{k}_{3}}{\rightleftharpoons}} \mathrm{IG}_{\mathrm{R}}$ \\
\hline IL-2 Induced production of IL-2 receptor & $\mathrm{IG}_{\mathrm{R}}$ & $\stackrel{\mathrm{k}_{5}}{\longrightarrow} \mathrm{IG}_{\mathrm{R}}+\mathrm{R}$ \\
\hline STAT5 activation & $\mathrm{IR}+\mathrm{S}$ & $\stackrel{\mathrm{k}_{6}}{\longrightarrow} \mathrm{IR}+\mathrm{S}^{*}$ \\
\hline STAT5 deactivation & $\mathrm{S}^{*}$ & $\stackrel{\mathrm{k}_{7}}{\longrightarrow} \mathrm{S}$ \\
\hline STAT5/gene binding & $\mathrm{S}^{*}+\mathrm{G}_{\mathrm{I}}$ & $\underset{\mathrm{k}_{9}}{\stackrel{\mathrm{k}_{8}}{\rightleftharpoons}} \mathrm{G}_{\mathrm{I}} \mathrm{S}^{*}$ \\
\hline IL-2/FasL gene binding & $\mathrm{I}+\mathrm{G}_{\mathrm{F}}$ & $\underset{\mathrm{k}_{11}}{\stackrel{\mathrm{k}_{10}}{\rightleftharpoons}} \mathrm{IG}_{\mathrm{F}}$ \\
\hline IL-2 Induced production of FasL & $\mathrm{IG}_{\mathrm{F}}$ & $\stackrel{\mathrm{k}_{12}}{\longrightarrow} \mathrm{IG}_{\mathrm{F}}+\mathrm{F}$ \\
\hline Caspase 8 activation & $\mathrm{F}+\mathrm{C}$ & $\stackrel{\mathrm{k}_{13}}{\longrightarrow} \mathrm{F}+\mathrm{C}^{*}$ \\
\hline Caspase 8 deactivation & $\mathrm{C}^{*}$ & $\stackrel{\mathrm{k}_{14}}{\longrightarrow} \mathrm{C}$ \\
\hline IL-2 degradation & I & $\stackrel{\delta_{1}}{\longrightarrow} \emptyset$ \\
\hline FasL degradation & $\mathrm{F}$ & $\stackrel{\delta_{2}}{\longrightarrow} \emptyset$ \\
\hline IL-2 Receptor degradation & $\mathrm{R}$ & $\stackrel{\delta_{3}}{\longrightarrow} \emptyset$ \\
\hline
\end{tabular}

\section{A. IL-2 Production and Ligand/Receptor Binding}

The mechanism of IL-2 binding to its corresponding receptor was obtained from [9]. However the two step reaction (IL2 binding to IL-2R $\alpha$ and association of the resulting complex with IL-2R $\beta$ and IL-2R $\gamma$ ) was simplified to only one single (IL-2 (I) binding to receptor(R)) event. IL-2 was assumed to diffuse rapidly within the model causing the system to function in the well-mixed regime. It has been shown through experimentation that there is an effective cooperativity of IL2 due to positive feedback on IL-2R $\alpha$ [10]. This complexity is described by IL-2 binding to the IL-2 receptor production gene $\left(G_{R}\right)$ which initiates production of the IL-2 receptor.

In addition, the cell population was assumed to be homogeneous. With these assumptions, we may model the effective rate of change of IL-2 concentration as the sum of the production/secretion of IL-2 from each cell. The production (and therefore secretion) of IL-2 is also down regulated as a function of activated STAT5 $\left(S^{*}\right)$. The mechanism of down regulation is described by activated STAT5 binding to the IL-2 production gene $\left(G_{I}\right)$, limiting IL-2 production [9].

\section{B. IL-2 Mediated STAT5 Activation}

From [9] we have identified that the binding of STAT5 onto the IL-2 receptor complex leads to its activation. STAT5 deactivation is also incorporated. In addition, it has been shown that activated STAT5 is essential for the proliferative response of T cells [9], [11], [12]. Here, we postulate that the cell proliferation rate is directly proportional to the activated STAT5.

\section{IL-2 Mediated Caspase8 Activation}

The Fas pathway is considered for explaining the IL-2 regulated mechanism of cell death [7], [13]. Fas signaling is initiated by oligomerization of the Fas receptor by FasL [14]. The cytoplasmic domain of Fas then binds the adapter 


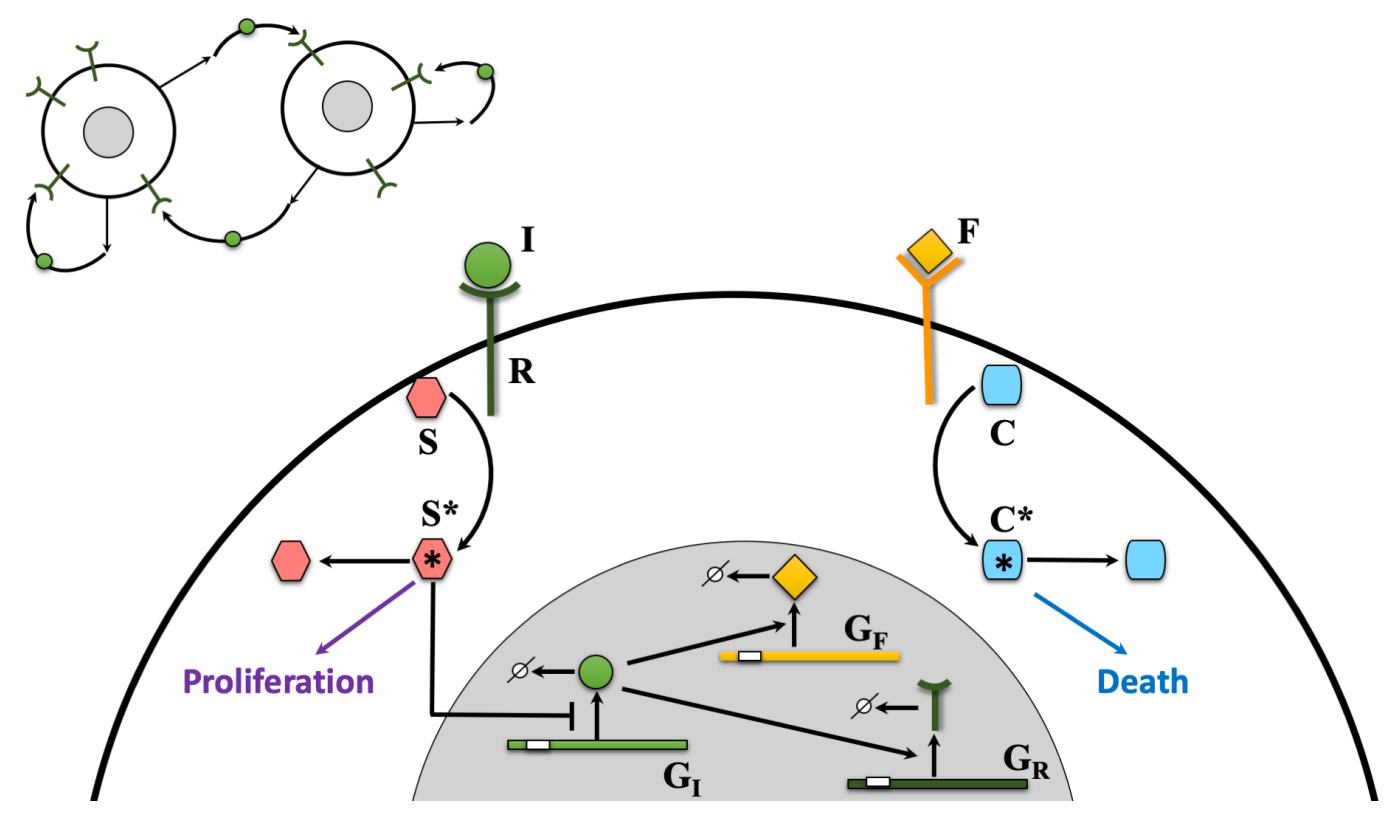

Fig. 1. Schematic of T-cell Intracellular Reactions. At the systems level (top left), IL-2 is secreted and detected by T-Cells which leads to change in population density. IL-2(I) binds to its receptor(R) and activates STAT5(S). FasL(F) initiates the activation of caspase8(C). Activated STAT5( $\left.S^{*}\right)$ and caspase $8\left(C^{*}\right)$ lead to T-cell proliferation and death respectively. Activated STAT5 also negatively regulates IL-2 production. IL-2 positively regulates the production of FasL and IL-2 Receptor. While STAT5 and caspase8 are conserved quantities, IL-2, FasL, and IL-2 receptor get degraded.

molecule FADD/MORT-1. This is followed by the binding FLICE/MACH-1 (caspase-8) [15]. The caspase is activated, leading to a cascade of catalytic activation of caspases that culminates in apoptosis. IL-2 increases transcription and surface expression of Fas ligand (FasL) [7]. Assuming Fas and FADD/MORT-1 are present in large amounts, we have simplified the multi-step reaction of FasL $(\mathrm{F})$ mediated caspase 8 (C) activation to one reaction. The mechanism of IL-2 induced FasL production is also included. We furthermore postulate that the cell death rate is directly proportional to the activated caspase8 $\left(C^{*}\right)$.

\section{INTEGRATED SYSTEMS LEVEL AND INTRACELLULAR Level Model of T-Cell Paradoxical Signaling}

The differential equations corresponding to the reactions in table II are presented below. The equations are derived using mass action kinetics. For the population dynamics, we apply an exponential growth equation which is the standard method employed in the quantitative analyses of mammalian cell proliferation in culture. This method is based on the implicit assumption that, within a cell population under study, all division events give rise to daughter cells that always divide [16].

\section{A. IL-2 Production and Ligand/Receptor Binding}

Equations (1) - (3) summarize IL-2 receptor binding dynamics, IL-2 production, and receptor production. Here, we include an environmental source $\left(I_{0}\right)$ of IL-2. If this source is not present the low/off population homeostasis state is equal to zero [4].

$$
\begin{aligned}
\frac{d I}{d t}= & -k_{1} \cdot I \cdot R+k_{2} \cdot I R-k_{3} \cdot I \cdot G_{R}+k_{4} \cdot I G_{R} \\
& -k_{10} \cdot I \cdot G_{F}+k_{11} \cdot I G_{F}+N \cdot \lambda \cdot G_{I} \\
& -\delta_{1} \cdot I+I_{0} \\
\frac{d R}{d t}= & k_{5} \cdot I G_{R}-k_{1} \cdot I \cdot R+k_{2} \cdot I R-\delta_{3} \cdot R \\
& \frac{d G_{R}}{d t}=-k_{3} \cdot I \cdot G_{R}+k_{4} \cdot I G_{R}
\end{aligned}
$$

\section{B. IL-2 Mediated STAT5 Activation}

Equations (4) - (7) summarize the mechanisms of IL2 mediated STAT5 activation and down regulation of IL-2 production by activated STAT5 $\left(\mathrm{S}^{*}\right)$ binding to the IL-2 gene $\left(G_{I}\right)$.

$$
\begin{gathered}
\frac{d G_{I}}{d t}=-k_{8} \cdot S^{*} \cdot G_{I}+k_{9} \cdot G_{I} S^{*} \\
\frac{d I R}{d t}=-k_{1} \cdot I \cdot R+k_{2} \cdot I R \\
\frac{d S^{*}}{d t}=k_{6} \cdot I R \cdot S-k_{7} \cdot S^{*} \\
-k_{8} \cdot S^{*} \cdot G_{I}+k_{9} \cdot G_{I} S^{*} \\
\frac{d S}{d t}=-k_{6} \cdot I R \cdot S+k_{7} \cdot S^{*}
\end{gathered}
$$

\section{IL-2 Mediated Caspase8 Activation}

Equations (8) - (10) summarize FasL mediated caspase8 activation and IL-2 induced FasL production.

$$
\begin{gathered}
\frac{d F}{d t}=k_{12} \cdot I G_{F}-\delta_{2} \cdot F \\
\frac{d C}{d t}=-k_{13} \cdot F \cdot C+k_{14} \cdot C^{*}
\end{gathered}
$$




$$
\frac{d C^{*}}{d t}=k_{13} \cdot F \cdot C-k_{14} \cdot C^{*}
$$

\section{Cell Population Dynamics}

The net growth of the cell population $(\mathrm{N})$ is defined as proliferation minus death rate. We have postulated the dependency proliferation rate on the concentration of activated STAT5 $\left(S^{*}\right)$ and the death rate on the concentration of activated caspase8 $\left(C^{*}\right)$. The differential equation for the exponential growth is summarized in Equation (11). In addition to the STAT5 and caspase 8 mediated rates, we include a constant(basal) proliferation rate $\left(\alpha_{0}\right)$.

$$
\frac{d N}{d t}=\left\{\left(\alpha \cdot S^{*}+\alpha_{0}\right)-\beta \cdot C^{*}\right\} \cdot N
$$

\section{Simplified COUPLED \\ INTRACELLULAR-POPULATION DYNAMIC EQUATIONS}

For simplicity, intracellular signaling reactions are assumed to reach steady state much more rapidly compared to cell division. Under these assumption we simplify Equations (1) $-(10)$.

\section{A. IL-2 Production and Ligand/Receptor Binding}

We assume the following intracellular reactions have reached steady state: 1) activated STAT5 binding IL-2 production gene 2) IL-2 binding IL-2 receptor production gene and 3) IL-2 binding to its receptor. We may write:

$$
\left.\begin{array}{l}
\frac{d I R}{d t}=0 \\
\frac{d G_{I}}{d t}=0 \\
\frac{d G_{R}}{d t}=0
\end{array}\right\} \Rightarrow \frac{d I}{d t}=N \cdot \lambda \cdot \frac{\frac{k_{9}}{k_{8}} G_{I t o t}}{S^{*}+\frac{k_{9}}{k_{8}}}-\delta \cdot I+I_{0}
$$

Here, $G_{\text {Itot }}$ is the total amount of IL-2 production gene. As discussed previously, activated STAT5 $\left(S^{*}\right)$ down-regulates IL-2 production which is represented mathematically by the repression Hill equation found through simplification of the steady state STAT5/gene binding reaction.

\section{B. IL-2 Mediated STAT5 Activation}

Assuming that: 1) IL-2 receptor dynamics 2) IL-2 binding IL-2 receptor production gene 3)IL-2/receptor binding and STAT5 activation reactions have reached steady state, we may write:

$$
\left.\begin{array}{l}
\frac{d R}{d t}=0 \\
\frac{d G_{R}}{d t}=0 \\
\frac{d I R}{d t}=0 \\
\frac{d S}{d t}=0 \\
G_{I} S^{*}<<S_{t o t}
\end{array}\right\} \Rightarrow S^{*}=S_{t o t} \cdot \frac{I^{2}}{I^{2}+A}
$$

$$
A=\frac{k_{7}}{k_{6}} \cdot \frac{k_{4}}{k_{3}} \cdot \frac{\delta_{3}}{k_{5} G_{R}}
$$

$S_{t o t}$ is the the total amount of STAT5. The Hill function represents the cooperative effect of IL-2 on activated STAT5 given positive feedback of the IL-2 receptor.

\section{IL-2 mediated Caspase8 activation:}

Assuming that caspase8 activation and FasL dynamics have reached steady state:

$$
\left.\begin{array}{l}
\frac{d F}{d t}=0 \\
\frac{d C^{*}}{d t} \approx 0
\end{array}\right\} \Rightarrow C^{*}=C_{t o t} \cdot \frac{I}{I+B} \approx \frac{C_{t o t}}{B} \cdot I
$$

Where:

$B=\frac{k_{14}}{k_{13}} \cdot \frac{k_{11}}{k_{10}} \cdot \frac{\delta_{2}}{k_{12} G_{F}}<<I$

Here, $C_{t o t}$ is the total amount of caspase8. The resulting expression derived from these assumptions is a cooperative relationship between activated caspase 8 and IL-2. If we assume that the apparent disassociation constant B is much larger that IL-2 concentration, we can approximate a linear relationship between activated caspase8 and IL-2.

\section{Cell Population Dynamics}

Substituting the expressions for activated STAT5 and caspase8 (found through the above steady state assumptions), we may write the rate of change of cell number $(\mathrm{N})$ as a function of IL-2:

$$
\begin{aligned}
& \frac{d N}{d t}=\left\{\left(\alpha S_{t o t} \frac{I^{2}}{\left(I^{2}+A\right)}+\alpha_{0}\right)-\beta \frac{C_{t o t}}{B} \cdot I\right\} N \\
& \text { V. ANALYSIS OF HoMEOSTATIC STABILITY AS A } \\
& \text { FUnCTION OF INTRACELLULAR PARAMETERS }
\end{aligned}
$$

\section{A. Net Growth Growth Rate as a function of IL-2}

A graphical representation of equation (15) is shown in Figure 2. The left blue curve represents the death rate which is linear with respect to IL-2 under previously explained assumptions. The slope is directly dependent on the total amount of caspase8 (which is a conserved quantity). The slope of the death rate is inversely dependent on coefficient B which is associated with the mechanisms of IL-2/FasL gene binding, activation of caspase 8 and production and degradation of FasL. If the slope is too steep (or not steep enough) equilibrium points will be eliminated. This will effectively eliminate bistability in the population dynamics.

The left purple curve of Figure 2 represents the proliferation rate by a Hill function with coefficient of 2 . It should be noted that to achieve bistability, a Hill coefficient of at least of one of the expressions for growth or death must be $>1$. The apparent dissociation constant of the Hill curve is represented by coefficient $A$. Coefficient $\mathrm{A}$ is associated with the mechanisms of IL-2/receptor gene binding, activation of STAT5 and production and degradation of IL-2 receptor. In Figure 2, the net growth rate vs IL-2 (right red curve) is the proliferation minus death rate and is at equilibrium

Where: 

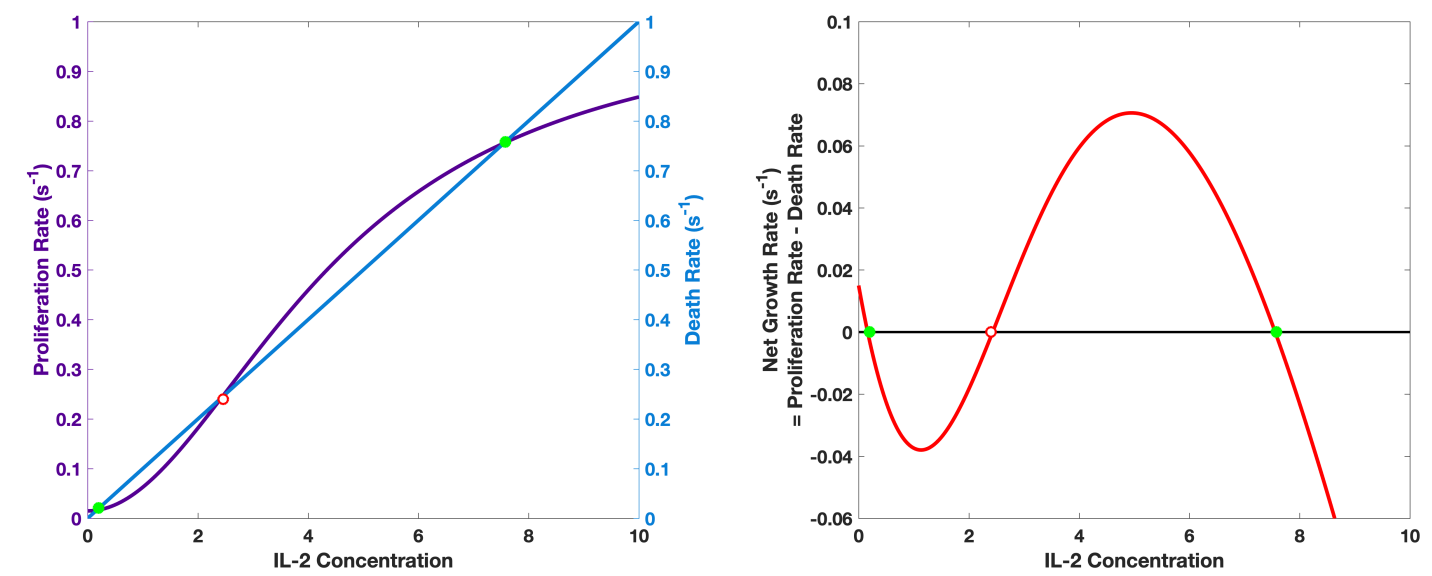

Fig. 2. Population Growth Rates as a function of IL-2 Cytokine. We assume that the proliferation rate (left purple curve) is proportional to activated STAT5. The cooperative effects of IL-2 during STAT5 activation and positive feedback of IL-2 receptor result in a Hill shaped curve. We assume that the death rate (left blue curve) is proportional to activated caspase8. The proliferation and death curves intersect at three points. The net growth rate (right red curve) is the proliferation minus death rate and is at equilibrium at three points.

at three points ( 2 stable filled green points and 1 unstable open red point). The bistable characteristics of this curve is representative of the paradoxical system. Above the threshold of IL-2 (represented by the unstable equilibrium point) the cell population will be driven towards the high/on population state. Below the threshold of IL-2 the cell population will be driven towards the low/off population state.

\section{B. Dependency of Net Growth Curve on Intracellular Mech- anistic Parameters}

To understand the dependence of net growth rate as a function of intracellular mechanistic parameters we examine Hill coefficient $A$. As previously discussed, coefficient $A$ is associated with the mechanisms of IL-2/receptor gene binding, activation of STAT5 and production and degradation of IL-2 receptor. More specifically, A is the product of 4 terms: 1) the disassociation constant associated with IL-2/receptor gene binding 2) the ratio of deactivation to activation rates of STAT5 3) the ratio of degradation to production rates of IL-2 receptor 4) the inverse of receptor gene concentration. Therefore smaller values of coefficient A signify larger forward rates of IL-2 induced production of IL-2 receptor, and increased STAT5 activation. As can

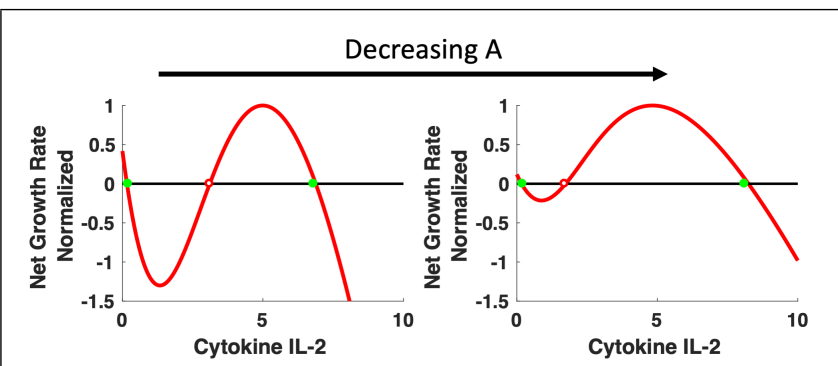

Fig. 3. Net growth (proliferation minus death rate) as a function of IL-2 for 2 paradoxical systems with varied parameters. As coefficient $A$ (described in the Hill equation of activated STAT5) decreases, the the middle(unstable signified by the open red circle) and largest(stable signified by the filled green circle) equilibrium points shift outward(away from each other). be seen from Figure 3, decreasing values of $A$ effectively shifts the threshold (middle unstable open red circle) and the high/on population state (largest stable filled green circle) outward, away from each other. This extends the range of initial conditions whose subsequent trajectories end up at the the high/on population state (basin of attraction). Whereas the basin of attraction reduces for the low/off population state.

\section{System Response to Disturbance}

For the analysis in the previous section we can infer increased stability to disturbances around the high/on steady state for decreased coefficient $A$. In Figure 4 an environmental disturbance causing removal of the same relative magnitude of IL-2 is introduced into paradoxical signaling systems with high and low coefficient $A$ at $\mathrm{t}=250$. In the system with larger coefficient $A$ (Figure 3 left), the cell population shifts below the unstable equilibrium to a regime with negative net growth (Figure $3 \mathrm{~A}$ left) resulting in the shift of IL-2 concentration to the lower equilibrium value (Figure 3B left) and the collapse of the cell population to the smaller homeostatic state (Figure 3C left).

In the system with smaller $A$ coefficient (Figure 3 right), the cell population remains in a regime with positive net growth (above the unstable equilibrium and within the basin of attraction) (Figure 3A right) . This results in the IL-2 concentration remaining robust to the disturbance (Figure 3B right) and the cell population is able to recover from the disturbance(Figure 3C right).

\section{CONCLUSIONS}

It has been shown that modeling the intracellular mechanisms involved in IL-2 regulated cell proliferation, cell death, and IL-2 production is sufficient to produce population dynamic characteristics of paradoxical signaling. Analysis of parameters revealed dependency of population homeostatic stability on the production of IL-2 receptor and activation of STAT5. Furthermore, the assumption that activated STAT5 is proportional to cell proliferation is sufficient to explain the 


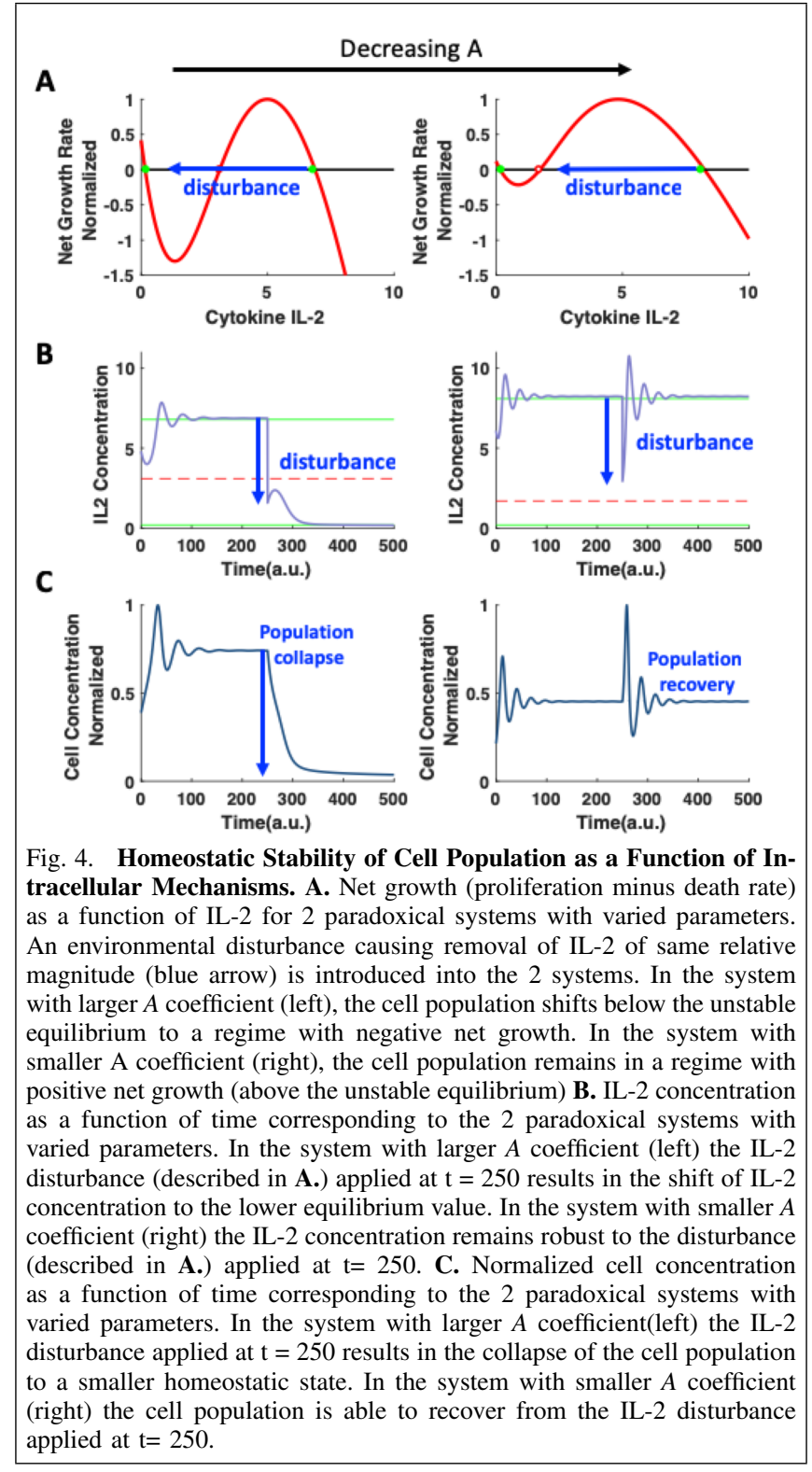

characteristic relationship between IL-2 and cell proliferation previously shown in [4]. The dynamics of caspase 8 was also shown to be consistent with the linear relationship between IL-2 and death rate. In the design of synthetic systems we can tune analogous parameters to get desired circuit functionality. In particular, depending on the applications it may be desired to have a unstable(easily switched between homeostatic states) paradoxical design or a stable(robust to disturbance) paradoxical design. Future work includes further analysis of parameters to test how net growth characteristics change. We may then propose analogous intracellular mechanisms which result in similar functional characteristics between signaling molecule growth and death for synthetic applications.

\section{ACKNOWLEDGMENT}

The authors would like to thank Cindy Ren, Yitong Ma, Mark Budde and Michael Elowitz for their insightful discussion.

\section{REFERENCES}

[1] L. You, R. S. Cox Iii, R. Weiss, and F. H. Arnold, "Programmed population control by cellcell communication and regulated killing," Nature, vol. 428, no. 6985, pp. 868-871, Apr. 2004. [Online]. Available: https://www.nature.com/articles/nature02491

[2] E. Distrutti, L. Monaldi, P. Ricci, and S. Fiorucci, "Gut microbiota role in irritable bowel syndrome: New therapeutic strategies," World Journal of Gastroenterology, vol. 22, no. 7, pp. 2219-2241, Feb. 2016. [Online]. Available: https://www.ncbi.nlm.nih.gov/pmc/articles/PMC4734998/

[3] A. N. Miliotou and L. C. Papadopoulou, "CAR T-cell Therapy: A New Era in Cancer Immunotherapy," Current Pharmaceutical Biotechnology, vol. 19, no. 1, pp. 5-18, 2018.

[4] Y. Hart, S. Reich-Zeliger, Y. E. Antebi, I. Zaretsky, A. E. Mayo, U. Alon, and N. Friedman, "Paradoxical signaling by a secreted molecule leads to homeostasis of cell levels," Cell, vol. 158, no. 5, pp. 1022-1032, Aug. 2014.

[5] O. Karin and U. Alon, "Biphasic response as a mechanism against mutant takeover in tissue homeostasis circuits," Molecular Systems Biology, vol. 13, no. 6, Jun. 2017. [Online]. Available: https://www.ncbi.nlm.nih.gov/pmc/articles/PMC5488663/

[6] J. Zhu and W. E. Paul, "CD4 T cells: fates, functions, and faults," Blood, vol. 112, no. 5, pp. 1557-1569, Sep. 2008.

[7] Y. Refaeli, L. Van Parijs, C. A. London, J. Tschopp, and A. K. Abbas, "Biochemical mechanisms of IL-2-regulated Fas-mediated T cell apoptosis," Immunity, vol. 8, no. 5, pp. 615-623, May 1998.

[8] Y. Hart and U. Alon, "The Utility of Paradoxical Components in Biological Circuits," Molecular Cell, vol. 49, no. 2, pp. 213-221, Jan. 2013. [Online]. Available: http://www.sciencedirect.com/science/article/pii/S1097276513000063

[9] O. Feinerman, G. Jentsch, K. E. Tkach, J. W. Coward, M. M. Hathorn, M. W. Sneddon, T. Emonet, K. A. Smith, and G. Altan-Bonnet, "Single-cell quantification of IL-2 response by effector and regulatory T cells reveals critical plasticity in immune response," Molecular Systems Biology, vol. 6, p. 437, Nov. 2010.

[10] N. Waysbort, D. Russ, B. M. Chain, and N. Friedman, "Coupled IL2Dependent extracellular feedbacks Govern two distinct Consecutive phases of CD4 T Cell activation," The Journal of Immunology, p. 1301575, 2013

[11] R. Moriggl, D. J. Topham, S. Teglund, V. Sexl, C. McKay, D. Wang, A. Hoffmeyer, J. van Deursen, M. Y. Sangster, K. D. Bunting, G. C. Grosveld, and J. N. Ihle, "Stat5 Is Required for IL-2-Induced Cell Cycle Progression of Peripheral T Cells," Immunity, vol. 10, no. 2, pp. 249-259, Feb. 1999. [Online]. Available: http://www.sciencedirect.com/science/article/pii/S1074761300800254

[12] N. Osinalde, H. Moss, O. Arrizabalaga, M. J. Omaetxebarria, B. Blagoev, A. M. Zubiaga, A. Fullaondo, J. M. Arizmendi, and I. Kratchmarova, "Interleukin-2 signaling pathway analysis by quantitative phosphoproteomics," Journal of Proteomics, vol. 75, no. 1, pp. $177-$ 191, Dec. 2011

[13] R. M. Siegel, F. K. Chan, H. J. Chun, and M. J. Lenardo, "The multifaceted role of Fas signaling in immune cell homeostasis and autoimmunity," Nature Immunology, vol. 1, no. 6, pp. 469-474, Dec. 2000.

[14] S. Nagata, "Apoptosis by death factor," Cell, vol. 88, no. 3, pp. 355365, Feb. 1997.

[15] M. P. Boldin, T. M. Goncharov, Y. V. Goltseve, and D. Wallach, "Involvement of MACH, a Novel MORT1/FADD-Interacting Protease, in Fas/APO-1- and TNF ReceptorInduced Cell Death," Cell, vol. 85, no. 6, pp. 803-815, Jun. 1996. [Online]. Available: https://www.cell.com/cell/abstract/S0092-8674(00)81265-9

[16] J. L. Sherley, P. B. Stadler, and J. S. Stadler, "A quantitative method for the analysis of mammalian cell proliferation in culture in terms of dividing and non-dividing cells," Cell proliferation, vol. 28, no. 3, pp. 137-144, 1995.

[17] J.-J. E. Slotine and W. Li, Applied nonlinear control. Prentice hall Englewood Cliffs, NJ, 1991, vol. 199, no. 1 